\title{
Inhibitory effect of miR-182-5p on retinal neovascularization by targeting angiogenin and BDNF
}

\author{
CHENYUE LI* ${ }^{*}$ HONGXUAN LIE* and WEIFENG SUN \\ Department of Ophthalmology, Changhai Hospital, Naval Medical University, Shanghai 200082, P.R. China
}

Received June 14, 2021; Accepted September 16, 2021

DOI: $10.3892 / \mathrm{mmr} .2021 .12577$

\begin{abstract}
Retinal neovascularization (RNV) is a type of serious vision-threating disease, commonly induced by hypoxia of ischemic retinopathy, which happens in various ocular diseases including diabetic retinopathy and retinopathy of prematurity. In clinical work, anti-VEGF therapy is the preferred strategy for treating RNV. However, not all cases are sensitive to anti-VEGF injection. It is urgent and necessary to develop novel targets for inhibiting neovascularization in ocular diseases. Angiogenin (ANG) and brain-derived neurotrophic factor (BDNF) are implicated in angiogenesis, although their regulation and effects in $\mathrm{RNV}$ remain to be elucidated. microRNA (miRNA) is a type of small non-coding RNA, which can modulate targets by degrading transcripts or inhibiting protein translation. In the present study, miRNA-mediated modulation of ANG and BDNF was explored in an oxygen-induced retinopathy mouse model and human retinal microvascular endothelial cells (HRECs) under hypoxia. The results showed that downregulation of miR-182-5p and upregulation of ANG and BDNF were found in vivo and in vitro. Overexpression of miR-182-5p suppressed the expression of ANG and BDNF significantly in HRECs under hypoxia. In addition, knockdown of ANG and BDNF by miR-182-5p transfection significantly improved hypoxia-induced HRECs dysfunctions, including enhancing cell viability, reducing cell migration and improved tube integrity. In conclusion, miRNA-dependent regulation on ANG and BDNF indicates a critical role in hypoxia-induced retinal microvascular response. miR-182-5p-based therapy can influence the expression of ANG and BDNF, which demonstrates the potential for treating RNV diseases.
\end{abstract}

Correspondence to: Professor Weifeng Sun, Department of Ophthalmology, Changhai Hospital, Naval Medical University, 168 Changhai Road, Shanghai 200082, P.R. China

E-mail: 13817834960@163.com

${ }^{*}$ Contributed equally

Key words: microRNA, angiogenin, brain-derived neurotrophic factor, retinal neovascularization, hypoxia

\section{Introduction}

The increasing occurrence of retinal neovascularization (RNV) threatens the quality of vision of individuals suffering from ocular neovascular diseases, for instance, proliferative diabetic retinopathy (PDR), retinal vein occlusions (RVO), retinopathy of prematurity (ROP) and wet age-related macular degeneration (wAMD) (1). Hypoxia occurs in these types of ocular disorders and is shown to be the key inducer in the development of RNV $(2,3)$. Hypoxia can stimulate diseased and dysfunctional cells to release non-physiological dose of angiogenic factors, thereby inducing morphological and functional alterations in the eye and even finally causing blindness (4). Current clinical treatment strategies, including intravitreally injection of anti-VEGF are sight-saving, but restricted by invasiveness, late intervention and high cost (5). Thus, it is urgent to advance a novel therapy to simultaneously protect neuronal and vascular functions in ischemic retinopathy diseases.

Angiogenin (ANG), a member of the pancreatic ribonuclease superfamily, is one of the most effective angiogenic factors in the body and has the ability to stimulate the vascular endothelial cells to promote cell proliferation and enhance tubular structure formation (6). ANG can accelerate the process of angiogenesis due to its specific capabilities as a transcription factor and a secreted protein. After being translated to the cytoplasm, ANG is instantly transported to the nucleus and then carries out angiogenic functions with angiogenesis inducers (e.g., VEGF) (7). Therefore, ANG may show a crucial role to induce angiogenesis in RNV and the molecular mechanism requires to be explored.

Brain-derived neurotrophic factor (BDNF), a humoral protein, can bind with tropomyosin receptor kinase $\mathrm{B}$ (TrkB) on nerve cells and subsequently activates growth of neurons (8). Previous studies report that BDNF signaling also promotes cells growth in addition to neural cells, such as vascular endothelial cells $(9,10)$. BDNF has been shown to be an angiogenic inducer similar to VEGF. For retina, a representative of neurovascular subunits, neurons and glial cells probably interact with blood vessels to regulate pathologic neovascularization by relieving growth factors and guidance cues (11). Thus, the potential role of BDNF in RNV is needed to be clearly elucidated.

MicroRNA (miRNA) is a highly conserved endogenous, small non-coding RNA and it can serve a role in regulating 
gene expression at the transcriptional or post-transcriptional level (12). It has been reported that miRNAs are enrolled in various types of activities and functions of endothelial cells, such as angiogenesis (13-15). A single miRNA can target hundreds of mRNAs, while each mRNA sustains various miRNA-response elements that can be targeted by different miRNAs. Therefore, different mRNAs can be modulated by a same miRNA. These RNA transcripts serve the part of competing endogenous RNAs (ceRNAs) (16). They can crosstalk with and co-regulate each other by competing for binding to shared miRNAs (17). Nevertheless, whether this modulatory mechanism of ceRNA happens between ANG- and BDNF-mediated angiogenesis in RNV remains to be elucidated. The present study hypothesized that ANG and BDNF expression is interdependently responsible for hypoxia through competition for a common miRNA in the development of RNV.

\section{Materials and methods}

Ethics statement. All animal procedures were examined and approved by The Animal Ethics Committee of Renji Hospital of Shanghai Jiaotong University (approval no. SHJT-MRJ-2020091). All surgeries were carried out under general anesthesia by sodium pentobarbital and best efforts were made to minimize the suffering of animals.

Oxygen-induced retinopathy (OIR) mouse model. Pregnant female C57BL/6J mice (weight, 22-25 g; age, 10 weeks old; $n=15$ ) were purchased from the Laboratory Animal Center of Renji Hospital. Mice were housed in standard plastic rodent cages with free access to food and water, and maintained in a controlled environment $\left(24^{\circ} \mathrm{C}, 12-\mathrm{h}\right.$ light/dark cycle). The mouse model of OIR exposed 7 -day-old (P7) mouse pups to $75 \pm 2 \%$ oxygen for 5 days (until P12). The animals were returned to room air for another 5 days until P17. The OIR model prepared by the above method can be used to study and describe the mechanisms of initial vessel loss (P7-P12), vascular regrowth (P12-P17) and neovascularization (P14-P17), respectively (18). Western blotting and PCR detection were carried out at P17. The mice were anesthetized with $2 \%$ sodium pentobarbital in a dose of $45 \mathrm{mg} / \mathrm{kg}$. Finally, the eyeballs were extracted, and the cervical spine of the mice were dislocated under anesthesia.

Cell culture and treatment. Human retinal microvascular endothelial cells (HRECs) were supplied by Angio Proteomie and cultured with endothelial cell medium including $5 \%$ fetal bovine serum (FBS) and 1\% endothelial cell growth supplement (ScienCell Research Laboratories, Inc.). HRECs were cultured in a humidified atmosphere with $37^{\circ} \mathrm{C}$ and $5 \% \mathrm{CO}_{2}$. For hypoxic stress, HRECs were seeded into a 30-mm dish (Corning Life Sciences) and cultured in a humidified atmosphere with $37^{\circ} \mathrm{C}$ and $5 \% \mathrm{CO}_{2}$; the medium was replaced when the cell confluence reached $\sim 80 \%$. Subsequently, HRECs were placed in a sealed and anaerobic workstation (Ruskin Technologies) at the atmosphere of $1 \% \mathrm{O}_{2}, 5 \% \mathrm{CO}_{2}$, $94 \% \mathrm{~N}_{2}, 90 \%$ humidity and $37^{\circ} \mathrm{C}$, as under hypoxic conditions for 48 or $72 \mathrm{~h}$.
Reverse transcription-quantitative $(R T-q) P C R$. Total RNA was extracted from HRECs ( $6 \times 10^{8}$ cells) exposed to hypoxia and retinal tissues by an Eastep Super Total RNA Extraction kit, supplied by Promega (Shanghai, China), according to the instructions of the kit. The concentration and purity of RNA were tested by a NanoDrop 2000c Spectrophotometer (Thermo Fisher Scientific, Inc.). For analysis of mRNAs, $1,000 \mathrm{ng}$ total RNA was reverse transcribed into cDNA by a Perfect Real Time RT Reagent kit, obtained from Takara Biotechnology Co., Ltd., in a $20 \mu \mathrm{l}$ reaction volume. The reverse transcription was performed at $37^{\circ} \mathrm{C}$ for $15 \mathrm{~min}, 85^{\circ} \mathrm{C}$ for $5 \mathrm{sec}$, then cooled to $4^{\circ} \mathrm{C}$ for maintenance. To investigate the expression of miRNA, 1,000 ng total RNA was polyadenylated and reverse transcribed using TransScript Green miRNA RT SuperMix, which was purchased from TransGen Biotech Co., Ltd. The reverse transcription was performed at $37^{\circ} \mathrm{C}$ for $60 \mathrm{~min}, 85^{\circ} \mathrm{C}$ for $5 \mathrm{sec}$, then decreased to $4^{\circ} \mathrm{C}$ for maintenance. SYBR Green Master Mix (Roche Diagnostics GmbH) was used for the PCR analysis of mRNAs. TransScript Green miRNA qPCR SuperMix (TransGen Biotech Co., Ltd.) was used to explore the expression of miRNA according to the manufacturer's instructions. The following thermocycling conditions were used for the qPCR: Initial denaturation at $94^{\circ} \mathrm{C}$ for $3 \mathrm{~min}$, followed by 40 cycles at $94^{\circ} \mathrm{C}$ for $20 \mathrm{sec}, 56^{\circ} \mathrm{C}$ for $20 \mathrm{sec}$ and $72^{\circ} \mathrm{C}$ for $20 \mathrm{sec}$. Melting curve analysis was conducted after every run by heating to $95^{\circ} \mathrm{C}$ to monitor the presence of unspecific products. The relative expression level of mRNAs or miRNAs was represented via the equation of $2^{-\Delta \Delta \mathrm{Cq}}(19)$. The primer sequences were: Human GAPDH: sense 5'-TGCACCACCAACTGCTTAGC-3', anti-sense 5'-GGCATGGACTGTGGTCATGAG-3'; Human ANG: sense 5'-ATGGCAACAAGCGCAGCATC-3', anti-sense 5'-CGGAC GACGGAAAATTGACTG-3'; Human BDNF: sense 5'-GTTT GTGTGGACCCCGAGTT-3', anti-sense 5'-GCAGCCTTCAT GCAACCAAA-3'; Mouse $\beta$-actin: sense 5'-GGCTGTATTCCC CTCCATCG-3', anti-sense:5'-CCAGTTGGTAACAATGCCATGT-3'; Mouse ANG: sense 5'-CATCCCAACAGGAAGGA AGGA-3', anti-sense 5'-ACCTGGAGTCATCCTGAGCC-3'; Mouse BDNF: sense 5'-AATGTCTGACCCCAGTGCCT-3', anti-sense 5'-ATGTTTGCGGCATCCAGGTA-3'.

Western blotting. The samples of cells or retinal tissues were lysed on ice for 60 min using Total Protein Extraction Buffer with protease inhibitor (TransGen Biotech Co., Ltd.) and followed by being sonicated $\left(20 \mathrm{KHz} ; 4^{\circ} \mathrm{C} ; 5 \mathrm{sec}\right.$; three times) referring to the manufacturer's instructions. Protein concentrations were determined using a BCA protein assay kit (Beyotime Institute of Biotechnology). The protein lysates $(15 \mu \mathrm{g})$ were obtained and electrophoresed on $10 \%$ SDS polyacrylamide gels and then transferred onto polyvinylidene difluoride membranes (MilliporeSigma). Subsequently, the samples were blocked with $5 \%$ skimmed milk for $1 \mathrm{~h}$ at room temperature. Primary antibodies against ANG (1:500; cat. no. DF6449; Affinity Biosciences, Ltd.), BDNF (1:500; cat. no. ab205067; Abcam) and $\beta$-actin (1:1,000; cat. no. AT0001; CMCTAG) were added at $4^{\circ} \mathrm{C}$ overnight. Membranes were then incubated with secondary antibodies (1:5,000; cat. nos. 32230 and 32260; Invitrogen; Thermo Fisher Scientific, Inc.) for $40 \mathrm{~min}$ at room temperature. Finally, an enhanced chemiluminescence (ECL) Plus kit (EMD Millipore) was used for visualization according 
to the manufacturer's instructions. The gray bands were analyzed by the ImageJ software (1.50i version; National Institutes of Health). The fold changes between different groups were demonstrated as the ratio of experimental group vs. the control group.

miRNA mimic transfection. HRECs growing at logarithmic growth stage were plated in a 6-well plate. On reaching $\sim 80 \%$ confluence, HRECs were transfected with 50 nmol miRNA mimic or scramble mimic using Lipofectamine ${ }^{\circledR}$ RNAiMAX transfection reagent (Invitrogen; Thermo Fisher Scientific, Inc.) according to the manufacturer's instructions. Following transfection for $5 \mathrm{~h}$ in a humidified atmosphere with $37^{\circ} \mathrm{C}$ and $5 \% \mathrm{CO}_{2}$, the cells were changed with fresh medium and cultured under hypoxia. After transfection for $48 \mathrm{~h}$, the cell samples were collected for subsequent mRNA detection. Following transfection for $72 \mathrm{~h}$, the samples were gathered and protein level investigated. The sequence of miR-182-5pmimic was 5'-UUUGGCAAUGGUAGAACUCAC ACU-3' and that of the scramblewas5'-CUCUCCGAACGUGUC ACGUTTUUC-3'; they were chemically synthesized by Shanghai GenePharma Co., Ltd.

Luciferase assay. TargetScan (http://www.targetscan. org/vert_72) was used to predict the targets of miRNA. The constructs of luciferase were constructed by combining oligonucleotides containing the wild-type (WT) or mutant (MUT) putative target sites of the ANG or BDNF 3'-untranslated region (3'-UTR) into the multiple cloning site of the pmirGLO vector (Promega Corporation). 293 cells were cotransfected with WT constructs, or MUT constructs, or vector and miRNA mimics or scramble miRNA using Lipofectamine ${ }^{\circledR} 2000$ (Invitrogen; Thermo Fisher Scientific, Inc.). At $48 \mathrm{~h}$ after transfection, luciferase detections were carried out by the Dual-Glo Luciferase Assay System (E2920; Promega Corporation) according to the manufacturer's instructions. Light emission was determined by the GloMax 96 Microplate Luminometer (Promega Corporation). Firefly luciferase activity was represented by normalizing to that of Renilla luciferase.

Cell viability assessment. MTS assay (Promega Corporation) was used to detect cell proliferation activity. HRECs were cultured under normal or hypoxic conditions with transfections as previously described and then plated at a density of $2 \times 10^{3}$ cells/well in a 96-well plate. Prior to evaluation, the medium was replaced by $100 \mu \mathrm{l}$ fresh medium and $20 \mu \mathrm{l}$ MTS was added into the samples. After culturing for $1 \mathrm{~h}$ in the incubator under normal oxygen, the absorbance of each samples was detected at $490 \mathrm{~nm}$.

Transwell assay. The migratory capacity of HRECs under hypoxic conditions was evaluated by the Transwell assay according to the manufacturer's protocols. In brief, $5 \times 10^{3}$ cells from each sample were placed in the top chambers of the Transwell chamber with $8.0-\mu \mathrm{m}$ pore polycarbonate membrane inserts (3422; Corning Life Sciences) and incubated with $200 \mu \mathrm{l}$ medium with $5 \%$ FBS. The bottom chambers of the Transwell were filled with $500 \mu \mathrm{l}$ medium containing $20 \%$ FBS. After incubating for $24 \mathrm{~h}$ in a humidified atmosphere with $37^{\circ} \mathrm{C}$ and $5 \% \mathrm{CO}_{2}$, HRECs that remained on the top chamber were removed and migrated cells were fixed by $4 \%$ paraformaldehyde for $20 \mathrm{~min}$ and then stained with $0.1 \%$ crystal violet solution for $10 \mathrm{~min}$ for observation, both at room temperature. The number of migrated cells were observed using a light microscope and counted in five fields randomly at x10 magnification. Quantitative analysis was conducted by Image J software (1.50i version; National Institutes of Health).

Tube formation analysis. The ability of angiogenesis of HRECs was detected by the tube formation assay. In brief, the 96-well plate was coated with $50 \mu 1$ of Matrigel Basement Membrane Matrix (BD Biosciences) and polymerized for $1 \mathrm{~h}$ at $37^{\circ} \mathrm{C}$. Subsequently, HRECs were added gently in the Matrigel-treated plate at the density of $8 \times 10^{3}$ cells per well with $100 \mu \mathrm{l}$ medium and cultured for $8 \mathrm{~h}$ at $37^{\circ} \mathrm{C}$. The angiogenic network of tubes was observed and images captured using a light microscope and counted in five fields randomly at x10 magnification. Quantitative analysis of tube formation was calculated by Image $\mathbf{J}$ software (1.50i version; National Institutes of Health).

Statistical analysis. All experiments in the present study were carried out at least three times independently. The data was demonstrated as the mean \pm standard deviation. Unpaired Student's t-test or one-way analysis of variance followed by Tukey's test were conducted by the GraphPad Prism 8.0 software (GraphPad Software, Inc.) to evaluate statistical differences. $\mathrm{P}<0.05$ was considered to indicate a statistically significant difference.

\section{Results}

Downregulated expression of miR-182-5p observed with the upregulation of ANG and BDNF in OIR mouse model. To predict the potential regulation of miRNA on ANG and BDNF, bioinformatics analysis was conducted. From the result shown in Fig. 1A, there are binding sites on the 3'-UTR of ANG and BDNF of a common miRNA, which is miR-182-5p. To explore the molecular mechanism of miR-182-5p on the regulation of ANG and BDNF in RNV, an OIR mouse model was firstly established. It was found that the expression level of miR-182-5p was inhibited in retinas of OIR (Fig. 1B), while the mRNA expression of ANG and BDNF was upregulated (Fig. 1C). Consistent with the transcriptional expression, the protein levels of ANG and BDNF were enhanced significantly (Fig. 1D and E). These observations revealed that miR-182-5p may regulate ANG and BDNF negatively and showed a critical function in the progression of RNV.

Hypoxia induces downregulation of miR-182-5p and overexpression of $A N G$ and BDNF in HRECs in vitro. To verify whether the observation in the OIR mouse model in vivo existed in vitro, HRECs were applied and exposed to hypoxia to mimic the circumstance of cells under RNV condition. Unsurprisingly, miR-182-5p was inhibited significantly by hypoxia in HRECs (Fig. 2A). In accordance with the changes in OIR retina, the mRNA expressions of ANG and BDNF were upregulated induced by hypoxia (Fig. 2B). In addition, 
A

\begin{tabular}{|c|c|c|}
\hline $\begin{array}{l}\text { Position 86-92 of ANG 3' UTR } \\
\text { hsa-miR-182-5p }\end{array}$ & $\begin{array}{l}5^{\prime} \\
3^{\prime}\end{array}$ & $\begin{array}{c}\text {... UGGUGGCAACAUUCAUUGCCAAG... } \\
\text { I I I I III } \\
\text { UCACACUCAAGAUGGUAACGGUUU }\end{array}$ \\
\hline $\begin{array}{l}\text { Position 1591-1597 of ANG 3' UTR } \\
\text { hsa-miR-182-5p }\end{array}$ & $5^{\prime}$ & $\begin{array}{c}\text {... UUAUUUAGAAAGUGGUUGCCAAU. . } \\
111111111 \\
\text { UCACACUCAAGAUGGUAACGGUUU }\end{array}$ \\
\hline $\begin{array}{l}\text { Position 2745-2751 of ANG 3' UTR } \\
\text { hsa-miR-182-5p }\end{array}$ & $5^{\prime}$ & $\begin{array}{c}\ldots \text { AAAAAAAAUGCAGUAAUUGCCAAU. } . . \\
111111\|1\| \\
\text { UCACACUCAAGAUGGUACGGUUU }\end{array}$ \\
\hline $\begin{array}{l}\text { Position 252-258 of BDNF } 3^{\prime} \text { UTR } \\
\text { hsa-miR-182-5p }\end{array}$ & $5^{\prime}$ & $\begin{array}{c}\text {... UGUGGUUUGUUGCCGUUGCCAAG... } \\
\text { IIIIIIII } \\
\text { UCACACUCAAGAUGGUACGGUUU }\end{array}$ \\
\hline
\end{tabular}

C

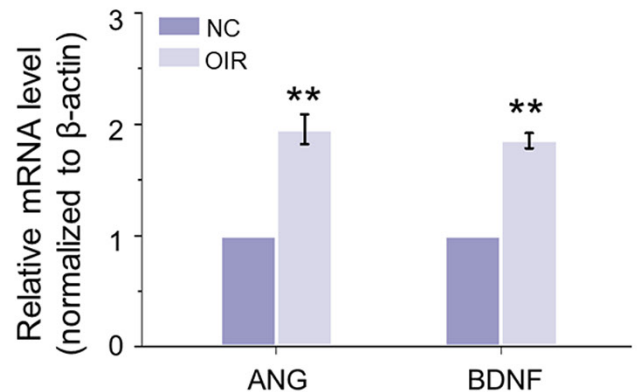

$\mathrm{B}$

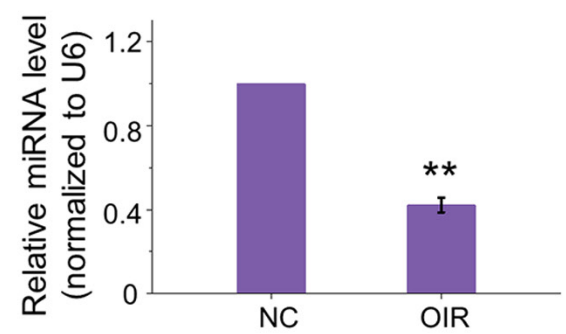

$\mathrm{E}$

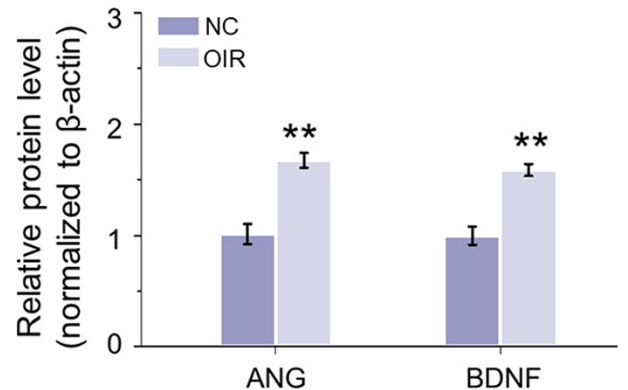

Figure 1. OIR-induced suppression of miR-182-5p and increased expression of ANG and BDNF. (A) The potential binding sites of miR-182-5p on 3'-UTR of ANG and BDNF predicted by TargetScan. (B) The reverse transcription-quantitative PCR analysis showed miR-182-5p was inhibited in retinas of OIR mouse compared with normal mouse. (C) The mRNA levels of ANG and BDNF were increased in OIR mouse retina, compared with normal mouse. (D) The representative grey bands of ANG and BDNF by western blotting. (E) The quantitation demonstrated the enhanced protein levels of ANG and BDNF in OIR mouse retinas. Means \pm SDs. ${ }^{* *} \mathrm{P}<0.01$ vs. the $\mathrm{NC}$ group $(\mathrm{n}=6)$. OIR, oxygen-induced retinopathy; $\mathrm{ANG}$, angiogenin; BDNF, brain-derived neurotrophic factor; miR, microRNA; NC, negative control.
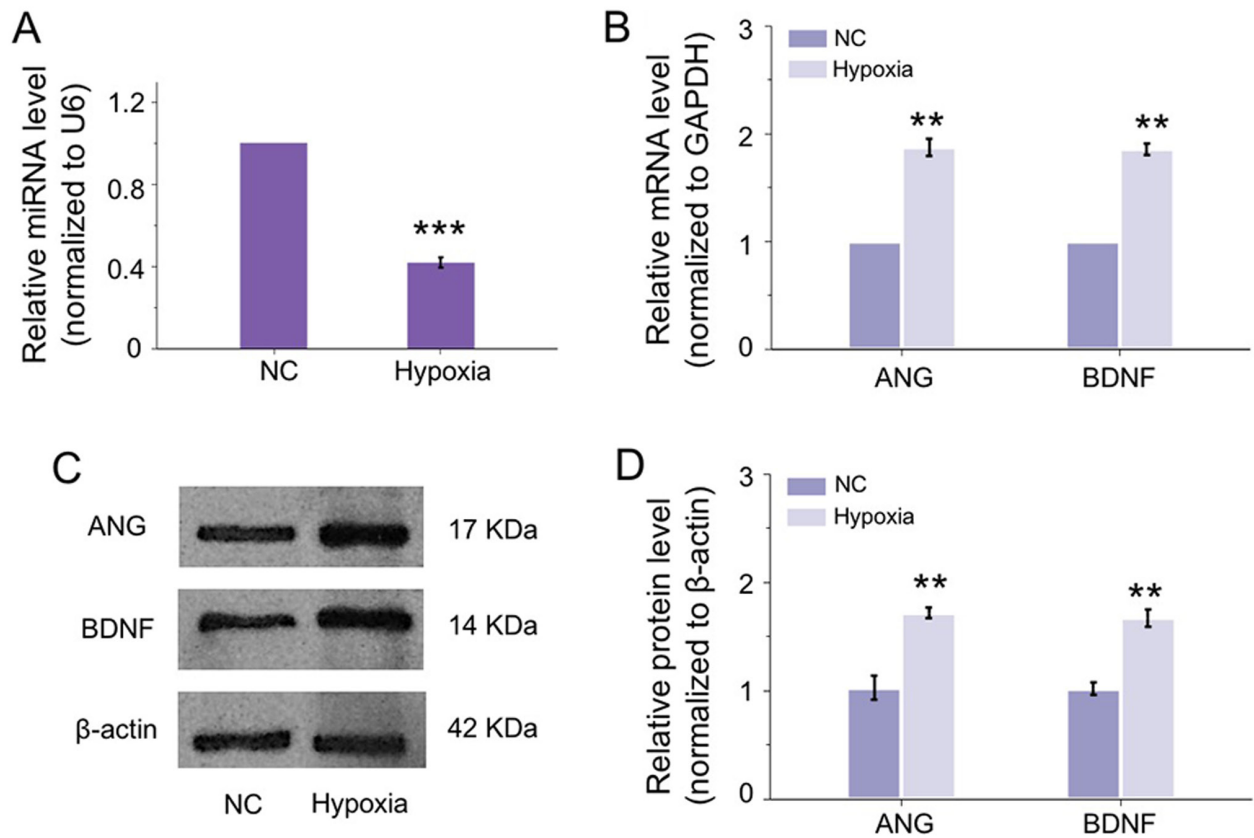

Figure 2. The expression levels of miR-182-5p, ANG and BDNF in HRECs under hypoxia. (A) miR-182-5p was downregulated by hypoxia in HRECs compared with normal condition detected by RT-qPCR analysis. (B) Through RT-qPCR performance, the transcriptional levels of ANG and BDNF were elevated compared with NC group in HRECs under hypoxic condition. (C) The western blots exhibited the increased expression of ANG and BDNF in hypoxic HRECs. (D) The quantitative analysis confirmed the significantly enhanced protein levels of ANG and BDNF caused by hypoxia in HRECs. ${ }^{* *} \mathrm{P}<0.01 ;{ }^{* * *} \mathrm{P}<0.001$ vs. the negative control group $(n=6)$. ANG, angiogenin; BDNF, brain-derived neurotrophic factor; miR, microRNA; HRECs, human retinal microvascular endothelial cells; RT-qPCR, reverse transcription-quantitative PCR; NC, negative control.

the translational expression of ANG and BDNF in HRECs was correspondingly increased under hypoxia (Fig. 2C and D). These results indicated the miR-182-5p may regulate the expression of ANG and BDNF in the development of $\mathrm{RNV}$ in vivo and in vitro. The molecular mechanism requires further exploration. 
A

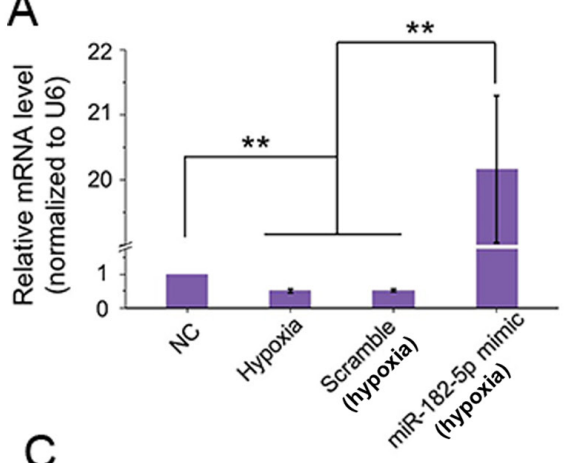

C
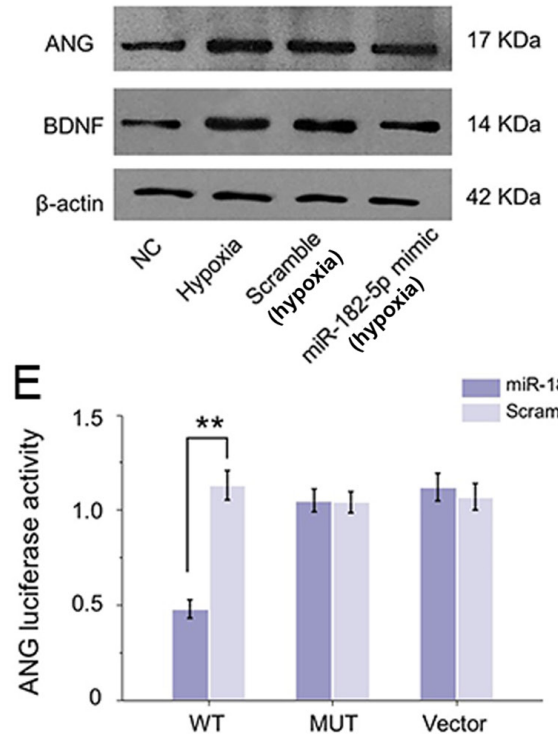

B
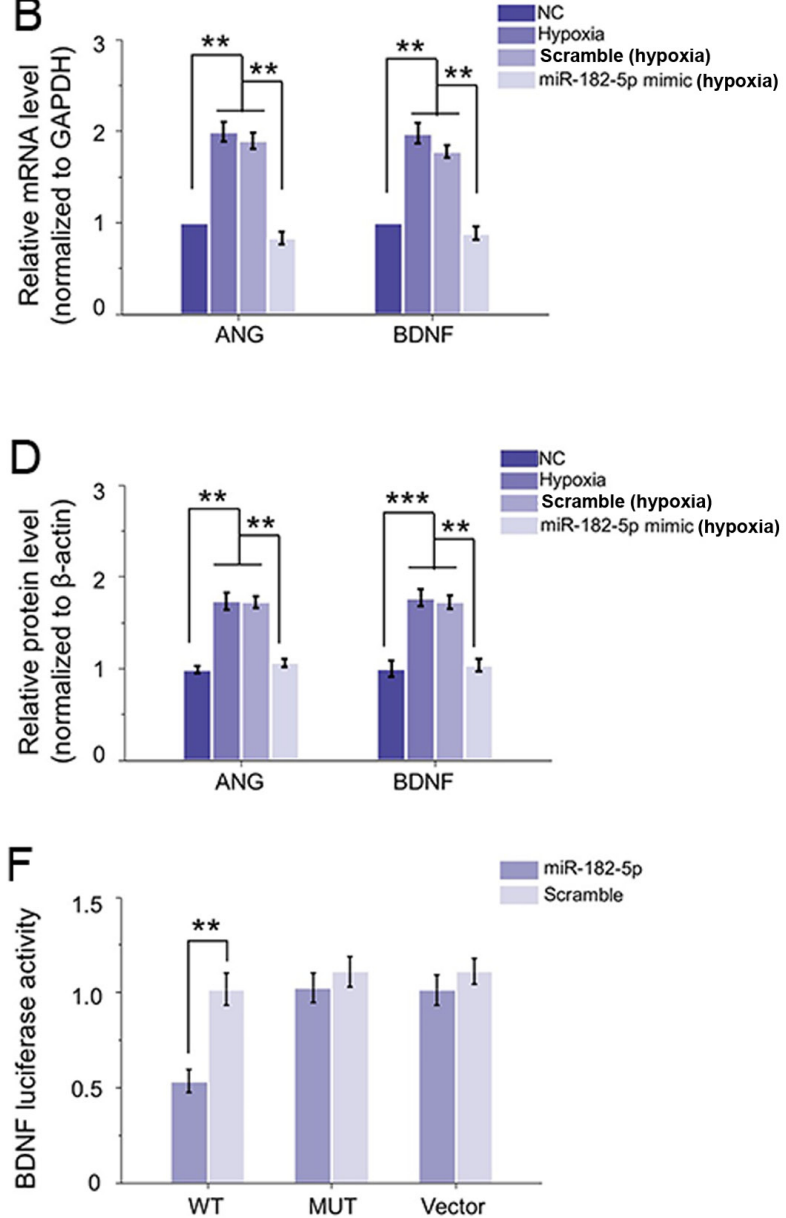

Figure 3. miR-182-5p can target ANG and BDNF directly. (A) Following transfection with miR-182-5p mimic, its expression was significantly elevated compared with hypoxic condition, while scramble did not induce any change. (B) Overexpression of miR-182-5p inhibited hypoxia-caused high mRNA levels of ANG and BDNF in HRECs, while scramble could not affect their expression. (C and D) Western blotting revealed the inhibitory effect of miR-182-5p on ANG and BDNF protein expression. (E and F) Luciferase activity with various reporters was detected in the presence or absence of miR-182-5p mimic in 293 cells. miR-182-5p mimic could effectively inhibit the luciferase activity of WT vectors of both ANG and BDNF compared with scramble transfection, neither MUT vector or vector showed a change with miR-182-5p mimic or scramble. ${ }^{* *} \mathrm{P}<0.01 ;{ }^{* * *} \mathrm{P}<0.001$ vs. relative control group ( $\mathrm{n}=6$ ). miR, microRNA; ANG, angiogenin; BDNF, brain-derived neurotrophic factor; HRECs, human retinal microvascular endothelial cells; WT, wild-type; MUT, mutant; NC, negative control.

miR-182-5p targets $A N G$ and BDNF directly. As the downregulation of miR-182-5p may be relevant to the upregulation of ANG and BDNF in HRECs and in OIR retina, the transfection interference experiment was performed to detect the regulatory effect of miR-182-5p on ANG and BDNF. With the transfection of miR-182-5p mimic, its expression level was increased $>20$-fold compared with either hypoxic or hypoxic with scramble transfection group. While scramble transfection did not result in any prominent change in miR-182-5p expression of HRECs under hypoxia compared with hypoxic group the two groups were inhibited compared with normal group (Fig. 3A). In addition, the hypoxia-induced increased mRNA expression and protein levels of ANG and BDNF in HRECs were clearly reduced with the upregulation of miR-182-5p (Fig. 3B-D). These revealed miR-182-5p could regulate the expression of ANG and BDNF in HRECs exposed to hypoxic condition. Subsequently, luciferase assay was performed to explore if miR-182-5p can target ANG and BDNF directly. By constructing the WT or MUT version of the predicted binding region in the ANG or BDNF 3'-UTR, the luciferase activity results demonstrated that co-transfection of 293 cells with 3'-UTR WT plasmid of ANG or BDNF and
miR-182-5p mimic caused a significant downregulation in luciferase activity compared with the scramble mimic. By contrast, miR-182-5p mimic indicated no inhibition on the luciferase activity of the MUT plasmid or vector (Fig. 3E and F). Therefore, ANG and BDNF could be suppressed by miR-182-5p directly in HRECs under hypoxic condition.

Inhibition of ANG and BDNF by miR-182-5p improves cell functions in HRECs exposed to hypoxia. The effects of miR-182-5p inhibiting ANG and BDNF on HRECs under RNV condition were then evaluated. Compared with normoxic condition, there was a significant effect on cell viability of HRECs under hypoxia. Knockdown of ANG and BDNF by miR-182-5p mimic transfection elevated the viability for $>48 \mathrm{~h}$. While the scramble transfection did not induce any significant change in cell viability compared with the hypoxic cells (Fig. 4A).

Transwell assays demonstrated that hypoxia can significantly enhance cell migration compared with that in normal groups (Fig. 4B). However, there was no significant difference in the results of migration in untransfected cells and 


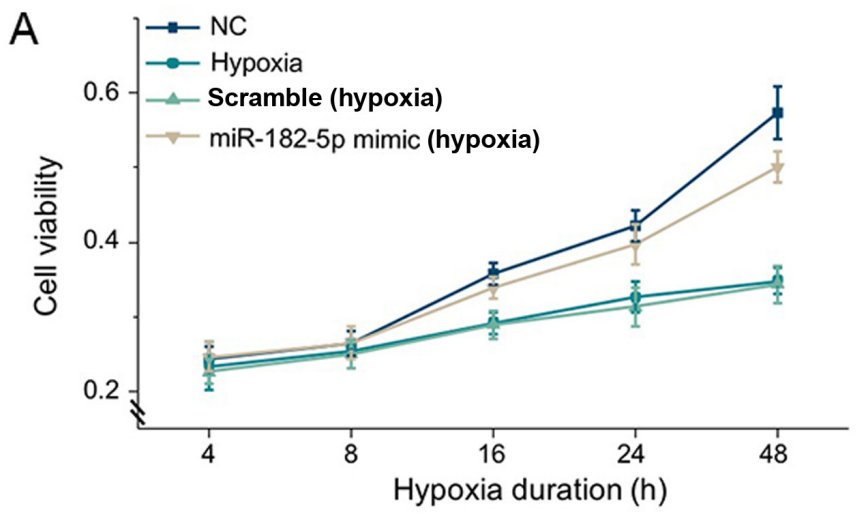

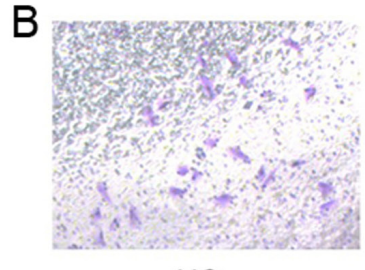

$\mathrm{NC}$

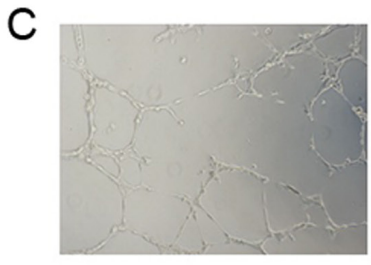

NC

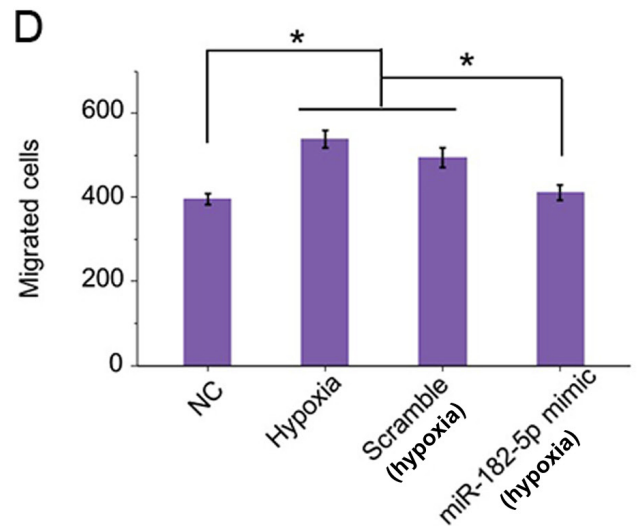

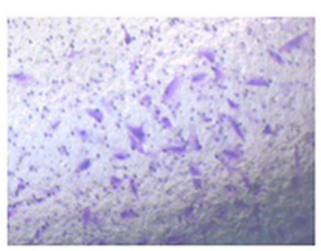

Hypoxia

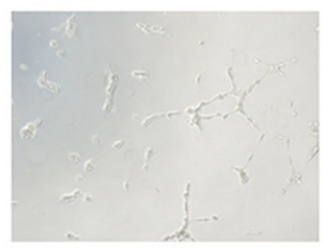

Hypoxia

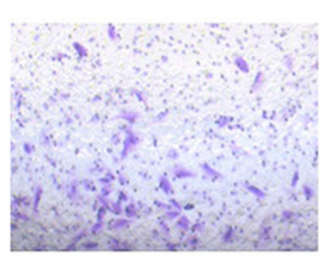

Scramble (hypoxia)

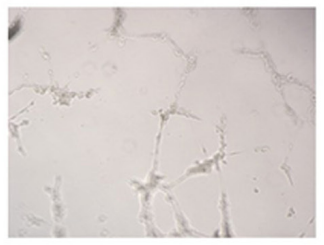

Scramble (hypoxia)

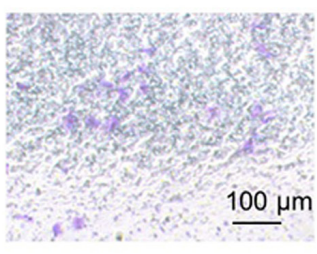

miR-182-5p mimic

(hypoxia)

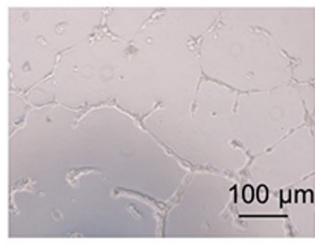

miR-182-5p mimic (hypoxia)

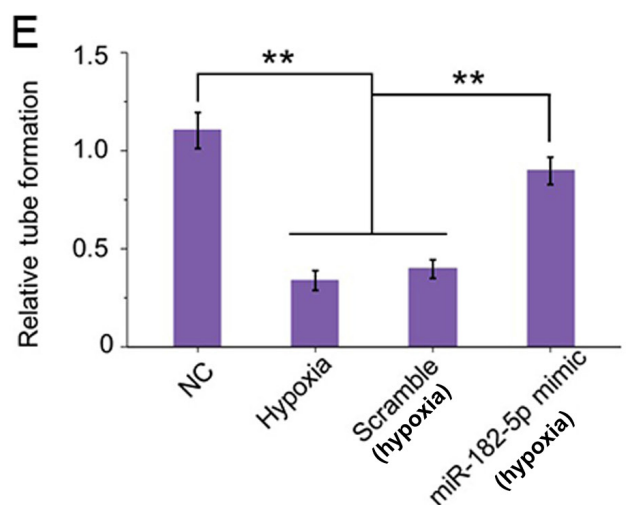

Figure 4. Effects of ANG and BDNF knockdown by miR-182-5p modulation on cell viability, cell migration and tube formation of HRECs under hypoxia (A) MTS assay showed changes in the cell viability of HRECs under hypoxia following upregulation of miR-182-5p over time. (B) Representative photomicrographs demonstrated the migrated cells in Transwell assays. Scale bar, 100- $\mu \mathrm{m}$. (C) Angiogenic ability of HRECs under normal or hypoxic conditions detected by tube network formation. Scale bar, $100-\mu \mathrm{m}$. Semi-quantitative analysis of (D) migrated cells and (E) relative tube formation ability. ${ }^{*} \mathrm{P}<0.05$; ${ }^{* *} \mathrm{P}<0.01$ vs. relative control group $(\mathrm{n}=6)$. ANG, angiogenin; BDNF, brain-derived neurotrophic factor; miR, microRNA; HRECs, human retinal microvascular endothelial cells; $\mathrm{NC}$, negative control.

scramble cells under hypoxia. In addition, under hypoxic condition, miR-182-5p overexpression protected against hypoxia-stimulated HRECs migration. The quantification also confirmed that the migrated cells were stimulated by hypoxia and could be significantly ameliorated by miR-182-5p transfection (Fig. 4D). Thus, inhibition of ANG and BDNF by miR-182-5p targeting under hypoxia reduced cell motility effectively.
In addition, Matrigel tube formation was evaluated in HRECs incubated under the same circumstance as previously described. As shown in Fig. 4C, hypoxic conditions could lead to a morphological change in HRECs and cause damage on the tube network formation compared with the normal group. In addition, ANG and BDNF intervention by miR-182-5p clearly enhanced the angiogenic ability of HRECs by inducing tube formation to 2-fold relative to that of the negative transfection 


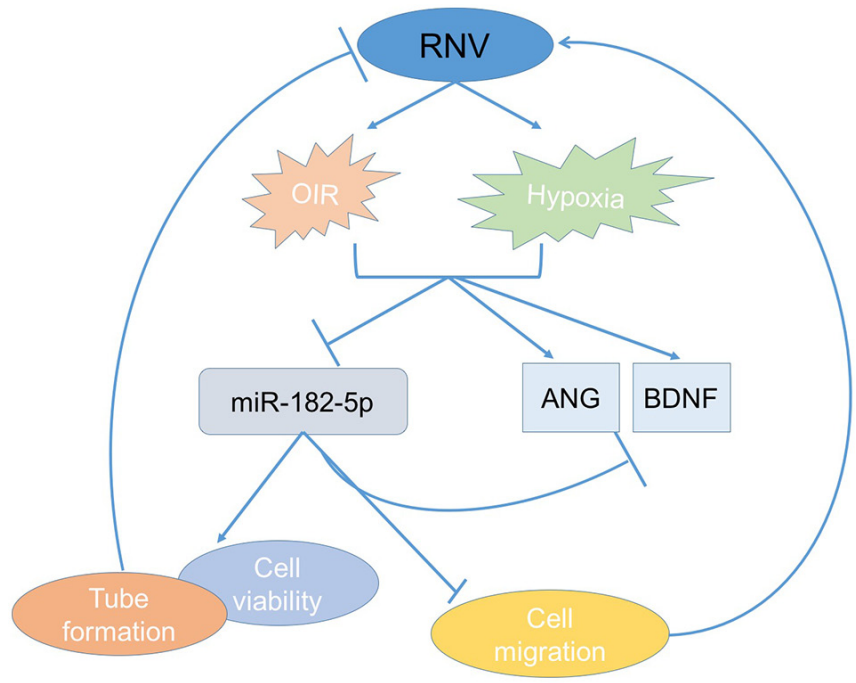

Figure 5. Overview of miR-182-5p on ameliorating retinal neovascularization by targeting ANG and BDNF. miR-182-5p protects HRECs functions by directly inhibiting ANG and BDNF under hypoxic condition or OIR model to ameliorate RNV. miR, microRNA; ANG, angiogenin; BDNF, brain-derived neurotrophic factor; HRECs, human retinal microvascular endothelial cells; OIR, oxygen-induced retinopathy; RNV, retinal neovascularization.

cells in hypoxic conditions (Fig. 4E). These findings indicated that knockdown of ANG and BDNF by miR-182-5p showed a protective function in HREC viability, motility, as well as tube integrity under RNV conditions. Correspondingly, inhibition of ANG and BDNF by miRNA regulation improved HREC morphology in RNV and might be a potential strategy for clinical treatment.

\section{Discussion}

Ocular diseases with RNV, including PDR, ROP and RVO, are commonly characterized by the pathological angiogenesis in the retina, finally leading to vision loss (20). Hypoxia is a crucial pathologic circumstance and stimulates the stimulation of proangiogenic factors supporting the formation of neovascularization in the progress of retinal neovascular diseases (21). Retinal angiogenesis is formed by the coordinate induction of a group of growth factor genes, especially VEGF. Therefore, the majority studies focus on finding alternative strategies for the development of anti-angiogenic VEGF inhibitors for curing retinal neovascularization $(22,23)$. However, a large number of preclinical investigations and clinical researches about retinal disorders indicate that limitations to anti-VEGF strategies may exist. Some patients do not respond to anti-VEGF treatment and some suffered from recurrences of neovascularization and bleeding. In addition, the secretion of growth factors besides VEGF may influence the response to anti-VEGF strategies in RNV diseases (24-26). Thus, it remains crucial for investigating the production of other proangiogenic factors and the breach of blood-retina barrier.

ANG is one of the most potential factors related to angiogenesis and it stimulates vessel formation by inducing the vessel endothelial and smooth muscle cells and activating a series of biological stages, including cell proliferation, migration and invasion, as well as tubular structures formation $(27,28)$. ANG also accelerates the degradation of basement membrane and extracellular matrix and promotes the migration capacity of the cells (29). Thus, the effects of ANG indicate its potential role in retinal microvascular endothelial cells in RNV. Angiogenesis is modulated by various types of factors, including growth factors and neurotrophins. BDNF is a type of neurotrophin and is recognized to regulate the nervous system in development, maintenance and plasticity (30). In addition, there are several types of cancer, such as multiple myeloma, breast cancer and thyroid cancer, that overexpress neurotrophins, including BDNF, which can be conducive to angiogenesis and tumor progression (31). Additionally, it is reported that BDNF displayed basic functions in non-neuronal tissues (32). It can be hypothesized that BDNF has a critical function in the progression of angiogenesis in RNV. Therefore, the interdependent crosstalk between ANG and BDNF in retinal angiogenesis deserves further exploration. The present study revealed a significant elevation of ANG and BDNF in vivo in the OIR model and in vitro in HRECs under hypoxic condition. To detect the modulatory mechanism on ANG and BDNF in RNV, non-coding RNA regulation stimulated was investigated.

miRNAs are small, non-coding RNAs that can bind to the 3'-UTR of target mRNAs to lead mRNA degradation or prohibit protein translation (14). More and more miRNAs have been identified in abnormal expression, indicating a crucial role in retinal neovascular diseases such as DR $(15,33,34)$. A previous study established the biogenesis, roles and functions of various miRNAs in the modulation of pathological ocular NV, revealing miRNAs as both biomarkers and therapeutic targets in vascular eye diseases (35). To compete for a common miRNA, protein-coding mRNAs may crosstalk with others without direct binding. By using TargetScan prediction, miR-182-5p may have a regulatory effect on ANG and BDNF. It was found that the expression of miR-182-5p was inhibited in vivo and in vitro and that overexpression of miR-182-5p could significantly restrain the expression levels of mRNA and protein of ANG and BDNF in vitro. In addition, the inhibitory effect of miR-182-5p on ANG and BDNF was confirmed by luciferase assay. These results demonstrated that miR-182-5p could target ANG and BDNF directly. Knockdown of ANG and BDNF by miR-182-5p upregulation protected HRECs against hypoxia-induced impairment, including enhancing cell viability, reducing cell migration and sustaining vascular tube network (Fig. 5). In addition, in a previous study, miR-182-5p also serves roles in angiogenesis in other vascular diseases (36). In colon cancer, miR-182-5p regulates tumorigenesis partially by regulating angiogenesis and lymphangiogenesis by targeting VEGF-C and therefore retarding ERK and AKT signaling pathways (36). This implied that miR-182-5p could regulate angiogenesis through different pathways. Thereby, miR-182-5p can be a potential therapeutic target to treat RNV.

The present study suggested that there was a cross-talk between ANG and BDNF mediated by the competition for miR-182-5p binding. Increased miR-182-5p expression resulted in a significant downregulation of ANG and BDNF and this regulation had a crucial function in the development of RNV including DR, RVO, ROP and other retinal diseases (e.g., age-related macular degeneration). miR-182-5p-based 
intervention not only affects the expression of growth factor-ANG, but also alters the level of neurotrophins-BDNF. Thus, miR-182-5p/ANG/BDNF cross-talk can have a clinical significance for the treatment of retinal neovascular disease.

\section{Acknowledgements}

Not applicable.

\section{Funding}

This study was funded by Changhai Hospital Teaching Research and Reform Project Fund in 2019 (grant no. CHJG2019012).

\section{Availability of data and materials}

The datasets used and/or analyzed during the current study are available from the corresponding author on reasonable request.

\section{Authors' contributions}

CL, HL and WS conceived and designed the experiments. CL and HL contributed to the acquisition of data. CL and HL analyzed and interpreted the data. CL, HL and WS contributed to drafting the article. All authors have revised the manuscript critically for important intellectual content. All authors read and approved the final manuscript. CL, HL and WS confirm the authenticity of all the raw data.

\section{Ethics approval and consent to participate}

The present study was approved by The Animal Ethics Committee of Renji Hospital of Shanghai Jiaotong University (approval no. SHJT-MRJ-2020-091). All surgeries were carried out under general anesthesia by sodium pentobarbital and best efforts were made to minimize the suffering of animals.

\section{Patient consent for publication}

Not applicable.

\section{Competing interests}

The authors declare that they have no competing interests.

\section{References}

1. Rajappa M, Saxena P and Kaur J: Ocular angiogenesis: Mechanisms and recent advances in therapy. Adv Clin Chem 50: 103-121, 2010.

2. Usui-Ouchi A, Aguilar E, Murinello S, Prins M, Gantner ML, Wright PE, Berlow RB and Friedlander M: An allosteric peptide inhibitor of HIF-1 $\alpha$ regulates hypoxia-induced retinal neovascularization. Proc Natl Acad Sci (USA) 117: 28297-28306, 2020.

3. Berkowitz BA: Hypoxia and retinal neovascularization. In: Retinal and Choroidal Angiogenesis Scientific Symposium, Nashville, TN, pp151-168, 2005.

4. Siemerink MJ, Augustin AJ and Schlingemann RO: Mechanisms of ocular angiogenesis and its molecular mediators. Dev Ophthalmol 46: 4-20, 2010
5. Antonetti DA, Klein R and Gardner TW: Diabetic retinopathy. N Engl J Med 366: 1227-1239, 2012.

6. Pavlov $\mathrm{N}$ and Badet J: Angiogenin: Involvement in angiogenesis and tumour growth. Bull Cancer 88: 725-732, 2001, (In French).

7. Kishimoto K, Liu S, Tsuji T, Olson KA and Hu GF: Endogenous angiogenin in endothelial cells is a general requirement for cell proliferation and angiogenesis. Oncogene 24: 445-456, 2005.

8. Allen SJ and Dawbarn D: Clinical relevance of the neurotrophins and their receptors. Clin sci (Lon) 110: 175-191, 2006.

9. Kermani P, Rafii D, Jin DK, Whitlock P, Schaffer W, Chiang A, Vincent L, Friedrich M, Shido K, Hackett NR, et al: Neurotrophins promote revascularization by local recruitment of TrkB+ endothelial cells and systemic mobilization of hematopoietic progenitors. J Clin Invest 115: 653-663, 2005.

10. Matsuda S, Fujita T, Kajiya M, Takeda K, Shiba H, Kawaguchi H and Kurihara $\mathrm{H}$ : Brain-derived neurotrophic factor induces migration of endothelial cells through a TrkB-ERK-integrin aVß3-FAK cascade. J Cell Physiol 227: 2123-2129, 2012.

11. Salis MB, Graiani G, Desortes E, Caldwell RB, Madeddu P and Emanueli C: Nerve growth factor supplementation reverses the impairment, induced by Type 1 diabetes, of hindlimb post-ischaemic recovery in mice. Diabetologia 47: 1055-1063, 2004.

12. Bartel DP: MicroRNAs: Target recognition and regulatory functions. Cell 136: 215-233, 2009.

13. Leung A and Natarajan R: Noncoding RNAs in vascular disease. Curr Opin Cardiol 29: 199-206, 2014.

14. Gong Q and Su G: Roles of miRNAs and long noncoding RNAs in the progression of diabetic retinopathy. Biosci rep 37: BSR20171157, 2017.

15. Gong Q, Xie J, Liu Y, Li Y and Su G: Differentially Expressed MicroRNAs in the Development of Early Diabetic Retinopathy. J Diabetes Res 2017: p1-10, 2017.

16. Giza DE, Vasilescu C and Calin GA: MicroRNAs and ceRNAs: Therapeutic implications of RNA networks. Expert Opin Biol Ther 14: 1285-1293, 2014.

17. Nitzan M, Steiman-Shimony A, Altuvia Y, Biham O and Margalit H: Interactions between distant ceRNAs in regulatory networks. Biophys J 106: 2254-2266, 2014.

18. Kim CB, D'Amore PA and Connor KM: Revisiting the mouse model of oxygen-induced retinopathy. Eye Brain 8: 67-79, 2016.

19. Livak KJ and Schmittgen TD: Analysis of relative gene expression data using real-time quantitative PCR and the 2(T (-Delta Delta C)) method. Methods 25: 402-408, 2001.

20. Selvam S, Kumar T and Fruttiger M: Retinal vasculature development in health and disease. Prog Retin Eye Res 63: 1-19, 2018.

21. Campochiaro PA: Molecular pathogenesis of retinal and choroidal vascular diseases. Prog Retin Eye Res 49: 67-81, 2015.

22. Lutty GA and McLeod DS: Development of the hyaloid, choroidal and retinal vasculatures in the fetal human eye. Prog Retin Eye Res 62: 58-76, 2018.

23. Rattner A, Williams $J$ and Nathans $J$ : Roles of HIFs and VEGF in angiogenesis in the retina and brain. J Clin Invest 129: 3807-3820, 2019.

24. Wallsh JO and Gallemore RP: Anti-VEGF-Resistant Retinal Diseases: A Review of the Latest Treatment Options. Cells 10: 1049, 2021.

25. Seah I, Zhao X, Lin Q, Liu Z, Su SZZ, Yuen YS, Hunziker W, Lingam G, Loh XJ and Su X: Use of biomaterials for sustained delivery of anti-VEGF to treat retinal diseases. Eye (Lond) 34: 1341-1356, 2020.

26. Michl M, Fabianska M, Seeböck P, Sadeghipour A, Haj Najeeb B, Bogunovic H, Schmidt-Erfurth UM and Gerendas BS: Automated quantification of macular fluid in retinal diseases and their response to anti-VEGF therapy. Br J Ophthalmol 0: 1-8, 2020.

27. Viallard C and Larrivée B: Tumor angiogenesis and vascular normalization: Alternative therapeutic targets. Angiogenesis 20: 409-426, 2017

28. Gao X and Xu Z: Mechanisms of action of angiogenin. Acta Biochim Biophys Sin (Shanghai) 40: 619-624, 2008.

29. Yao X, Li D, Xiong DM, Li L, Jiang R and Chen JX: A novel role of ribonuclease inhibitor in regulation of epithelial-to-mesenchymal transition and ILK signaling pathway in bladder cancer cells. Cell Tissue Res 353: 409-423, 2013.

30. Numakawa T, Richards M, Adachi N, Kishi S, Kunugi H and Hashido K: MicroRNA function and neurotrophin BDNF. Neurochem Int 59: 551-558, 2011. 
31. Pearse RN, Swendeman SL, Li Y, Rafii D and Hempstead BL: A neurotrophin axis in myeloma: TrkB and BDNF promote tumor-cell survival. Blood 105: 4429-4436, 2005.

32. Garrido MP, Torres I, Vega M and Romero C: Angiogenesis in Gynecological Cancers: Role of Neurotrophins. Front Oncol 9: 913, 2019.

33. Gong Q, Li F, Xie J and Su G: Upregulated VEGF and Robo4 correlate with the reduction of miR-15a in the development of diabetic retinopathy. Endocrine 65: 35-45, 2019.

34. Gong Q, Xie J, Li Y, Liu Y and Su G: Enhanced ROBO4 is mediated by up-regulation of HIF-1 $\alpha / \mathrm{SP} 1$ or reduction in $\mathrm{miR}-125 \mathrm{~b}-5 \mathrm{p} / \mathrm{miR}-146 \mathrm{a}-5 \mathrm{p}$ in diabetic retinopathy. J Cell Mol Med 23: 4723-4737, 2019.
35. Liu CH, Huang S, Britton WR and Chen J: MicroRNAs in Vascular Eye Diseases. Int J of Mol Sci 21:649.

36. Yan S, Wang H, Chen X, Liang C, Shang W, Wang L, Li J and $\mathrm{Xu}$ D: MiR-182-5p inhibits colon cancer tumorigenesis, angiogenesis, and lymphangiogenesis by directly downregulating VEGF-C. Cancer Lett 488: 18-26, 2020.

(i) (9) This work is licensed under a Creative Commons Attribution-NonCommercial-NoDerivatives 4.0 International (CC BY-NC-ND 4.0) License. 